Адрес статьи / To link this article: http://cat.itmo.ru/ru/2021/v6-i2/276

\title{
Бенджамин Йориссен. Структурная этнография виртуальных миров (перевод с немецкого)
}

\author{
А.Н. Липов \\ Институт философии РАН (сектор эстетики), Российская федерация \\ antolip@yandex.ru
}

\begin{abstract}
Перевод выполнен по изданию: Benjamin Jörissen. Strukturale Ethnografie Virtueller Welten // Grell P., Marotzki W., Schelhowe H. (eds). Neue digitale Kultur- und Bildungsräume. 2010. Berlin. Springer Nature. P. 119-143. DOI: 10.1007/978-3-531-91917-1_8
\end{abstract}

\begin{abstract}
Аннотация: Существование так называемых «виртуальных миров» широко известно со времен серии публикаций в СМИ о виртуальной цифровой платформе «Вторая жизнь» («Second Life»). B настоящей публикации, представляющей собой перевод с немецкого языка научной статьи, предпринимается попытка анализа механизмов создания, распространения, функционирования и развития в культуре, онлайн-сообществах, виртуальных цифровых пространствах новейших цифровых технологий, позволяющих проектировать высокотехнологические виртуальные среды и программные продукты с последующим их внедрением и асимиляцией в общественную среду, создания новой карты социальных миров, гибридной виртуальнореальной экономики и виртуально-социальных арен «реальной жизни».
\end{abstract}

Ключевые слова: виртуальная цифровая культура, виртуальная среда, виртуальные миры, онлайн-сообщества, структурная этнография, виртуальная платформа «Second Life», технология «Аватар», запрограммированные цифровые объекты, интерактивные алгоритмы, «Эффект Протеуса», цифровая общественная среда и функциональность.

Существование так называемых «виртуальных миров» широко известно со времен шумихи в СМИ вокруг сетевой платформы «Второй жизни» («Second Life»). Несмотря на то, что виртуальные ролевые вселенные, такие как «World of Warcraft (с англ. - «Мир военного ремесла», прим. пер.), являются гораздо более заметным феноменом, если смотреть на них с точки зрения чистого числа пользователей, существует большое количество предложений, с растущей тенденцией к более молодым целевым группам, особенно школьникам [1].

В начале 2009 года институт рыночных исследований «KZERO» зарегистрировал более сорока трех виртуальных миров для лиц в возрасте от 8 до 13 лет, несколько меньшее количество - для лиц в возрасте от 15 до 25 лет, а также отдельные предложения для пожилых людей, в том числе и упомянутую выше виртуальную цифровую платформу «Вторая жизнь», пользователям которой в среднем около 35 лет (для этих и следующих данных: «KZERO», 2009). 
Поразительно, что около половины этих миров находятся в пробной фазе, что свидетельствует о том, что в этой области наблюдается большая активность развития. В 2007 году только медиа-группа «Никелодеон» (Nickelodeon», с анл. - «автоматический проигрыватель», прим. пер.) инвестировала 100 миллионов долларов США в развитие сети виртуальных миров (на основе возрастных этапов) (Barnes, 2007).

Среди виртуальных миров, известных в Германии, можно назвать следующие: «Барбидевочки» (17 млн аккаунтов, из которых 5 млн новых с начала 2008 года), «Поптропика» (40 млн аккаунтов, из которых 20 млн новых с осени 2008 года); «Хаббо Отель» (124 млн аккаунтов, из которых 35 млн новых с начала 2008 года), мир виртуальных чатов «IMVU» (30 млн аккаунтов, из которых 10 млн новых с осени 2008 года) и «Second Life» (17 млн аккаунтов, из которых 4 млн новых с 2008 года). Для немецкоязычных стран следует также упомянуть виртуальный детский виртуальный мир «Панфу» с более чем 4 миллионами аккаунтов (отчет «Панфу») [2].

Несмотря на то, что, например, такие виртуальные ролевые вселенные, как, например, «Мир военных кораблей» («World of Warcraft») и подобные ей, являются гораздо более заметным феноменом, с точки зрения числа пользователей, как мы уже отметили ранее, существует большое число подобных организованных предложений с растущей тенденцией к их увеличению, особенно для младших целевых групп и школьников.

В связи с этими событиями возникает вопрос об образовательной ценности виртуальных миров уже хотя бы потому, что это не совсем новое явление само по себе. Различие между Виртуальными мирами и настоящими Виртуальными игровыми мирами может быть определено формальными особенностями игры, такими как наличие явных или подразумеваемых игровых целей и правил.

Виртуальные миры не имеют предопределенной цели действия, повествовательной последовательности, не имеют определенной топологии в смысле мест, функциональных для определенных аспектов игры и т.д. У них также нет правил игры, но, как и у любого онлайнсообщества, у них существуют социальные правила поведения. И виртуальные миры вполне могут содержать разнообразные встраиваемые игры.

Однако, это не превращает их в игру больше, чем флэш-игры в веб-браузере во всемирной паутине, или в социальных сетях, в которые они интегрированы [2]. Некоторые примеры видеоматериалов собраны по адресу http://www.youtube.com/user/joeriben; (выборочный), а список виртуальных миров можно найти по адресу: http://www.diigo.com/list/benjamin/virtualworlds [3].

Соответственно, исследовательская ситуация такова, что виртуальные миры или их аспекты, особенно телесные представления виртуальных миров, так называемые «аватары», уже некоторое время находятся в центре внимания: это, конечно, относится, прежде всего, к компьютерным наукам (Bormann, 1994), но также и к культурным исследованиям (Featherstone / Burrows, 1995), наукам о коммуникации (Steurer, 1996; Hillis, 1999), экспериментальной социальной психологии (Bente / Krämer/Petersen, 2002) и, наконец, к междисциплинарным антологиям (Schröder, 2002a; Schröder / Axelsson, 2006).

Предлагаемый здесь подход находится в контексте структурного образования в области средств массовой информации (Jörissen / Marotzki, 2009). Этот подход объединяет идею теории структурного образования (Marotzki, 1990) со структурным анализом медиа так, что может быть эмпирически задействован образовательный потенциал медиа-архитектур. Поэтому образование здесь понимается в более узком, подлинно образовательно-теоретическом смысле, как преобразование структурных моделей отношений между собой и миром.

Таким образом, вопрос заключается в том, предлагают ли, и в какой степени, структурные элементы виртуальных миров некий потенциал для процессов формирования и трансформации идентичности, для развития ориентировочного знания, а значит, и для переосмысления собственного образа мира и себя. При этом я хотел бы излагать материал здесь таким образом, чтобы в рамках трех уровней анализа, которые должны быть названы сразу же, были показаны соответствующие внутренние структуры, а затем они были бы обсуждены с точки зрения теории образования [4]. 
Виртуальные миры - это, по меньшей мере, потенциально очень сложные сущности. В них встречается множество различных участников из различных социальных и культурных контекстов, где они присваивают себе одну или несколько идентичностей (в форме аватаров), знакомятся, формируют группы, общаются, играют, сотрудничают друг с другом, и при этом часто создают множество новых форм личного и социального опыта. Антропологическое исследование Тома Бёллсторфа, который в течение нескольких лет исследует «Вторую Жизнь» под наблюдением участников, впечатляюще демонстрирует, в какой степени виртуальный мир может стать живым миром и местом биографических переживаний [5]. Различие между виртуальными мирами и настоящими виртуальными игровыми мирами может быть определено формальными особенностями игры, такими как наличие явных или неявных игровых целей и правил.

Каждый виртуальный мир, в соответствии со своими технологическими и техносоциальными базовыми структурами раскрывает свои сложные законы и тем самым открывает весьма специфические возможности для переживания, которые, как правило, существенно отличаются от других виртуальных миров. Одним из способов эмпирического разложения этих сложных структур было бы наблюдение за участниками в полевых условиях, как это делал Том Бёллсторф или ранее Т. Л. Тейлор (T. L. Taylor, 2002).

Другой путь, который является более осуществимым с точки зрения исследовательской прагматичности, поскольку с учетом большого количества и динамики развития он занимает значительно меньше времени, - это структурный анализ, введенный Винфридом Мароцки (Winfried Marotzki, 2003) для анализа онлайн-сообществ. Структурные категории позволяют уменьшить сложность и, в то же время, создают возможности для перекрестного сравнения. Прежде всего, как отмечалось выше, они позволяют дифференцированно обсуждать связи теории образования в контексте отдельных структурных областей.

Виртуальные миры можно рассматривать как особую форму онлайн-сообществ в социографическом плане, поскольку они могут быть описаны как структура общественных и частных площадок для целей коммуникации и взаимодействия. Однако сама по себе виртуальная среда не может быть сведена к функциям онлайн-сообщества. Поскольку виртуальные среды открыты для множества других областей применения, от научно-технического симулятора до компьютерного искусства и вышеупомянутой ролевой онлайн-игры, то очевидно анализировать их как отдельную структурную область.

Тогда связь между этими двумя областями лежит, например, в имитируемой пространственности арен, которые, таким образом, становятся «доступными» виртуальными местами и пространствами социального взаимодействия. Аватар аналитически отделен от этой виртуальной среды, хотя это и не является само собой разумеющимся, поскольку аватары, как правило, основаны на той же технологической и графической основе, что и виртуальный мир, в котором они появляются.

С одной стороны, постоянно ведется работа по описанию аватаров с помощью стандартизации, с целью их возможного использования в различных виртуальных средах таким образом, чтобы аватарская идентичность существовала в различных контекстах и относительно автономно по отношению к ним [6]. С другой стороны, аватары могут использоваться и вне виртуальных миров (например, «Weblin»-аватары), расположенные в веб-браузере оригинальном программном приложении, позволяющего общаться с людьми, посещающими ту же веб-страницу, где ваша личность в чате - полностью анимированный аватар [7].

Более важными, чем эти технические аспекты, являются особые отношения между аватаром и его владельцем или пользователем. Не случайно существуют исследования аватара, которые лишь незначительно учитывает аспект виртуальной среды (Petersen / Bente / Krämer, 2002; Schroeder, 2002a; Bailenson / Beall, 2005; Yee, 2007). Сложные и ярко выраженные, с образовательной точки зрения, эффекты отношений между аватаром и пользователем нельзя отнести к проектированию виртуальной среды или к функциональным возможностям сообщества.

Так, например, «Gravatar.com» - это широко распространенный сервис, позволяющий динамически интегрировать свой собственный «аватар» в разных местах WWW (например, рядом с вашими собственными комментариями в других блогах и т.д.). При этом эффект отношений между аватаром и пользователем, с точки зрения образования, не может быть отнесен ни к

Культура и технологии, Том 6, № 2 
дизайну виртуальной среды, ни к функциям сообщества. Как будет подробно показано ниже, они представляют собой самостоятельный и центральный аспект виртуальных миров, означающим, что следующие три структурные области виртуальных миров можно отличить друг от друга:

1. Виртуальная среда

2. Технология «Аватар»

3. Общественная среда и функциональность

В качестве четвертой структурной области, которую не следует включать в данный список, можно рассматривать социальные арены «реальной жизни», т.е. области вне виртуального мира, которые, тем не менее, их пересекают. Классическим примером этого была бы реальная экономика, которая одновременно действует как валюта внутри мира и может быть обменена на другие валюты за его пределами. Валюта во «Второй жизни» - «Линден-доллар» онлайнсообщества как раз и является такой валютой. Это дает возможность людям работать в «онлайнсообществах».

В случаe c «Second Life» гибридная виртуально-реальная мировая валюта также включает в себя множество других социальных арен, которые в незначительно степени или вообще не важны без экономических связей. Так как эта область не исследуется систематически (поскольку она, во-первых, выходит за рамки данного очерка, а во-вторых, представляет собой особый случай нескольких виртуальных миров), карта социальных миров и арен во «Второй Жизни» создает впечатление потенциального разнообразия и сложности этой четвертой структурной области по отношению к методологическим основам применяемого здесь «Ситуационного анализа» (Clarke, 2005). (см.: рис. 1; о методологическом фоне применяемого здесь «Ситуационного анализа»: Clarke, 2005).

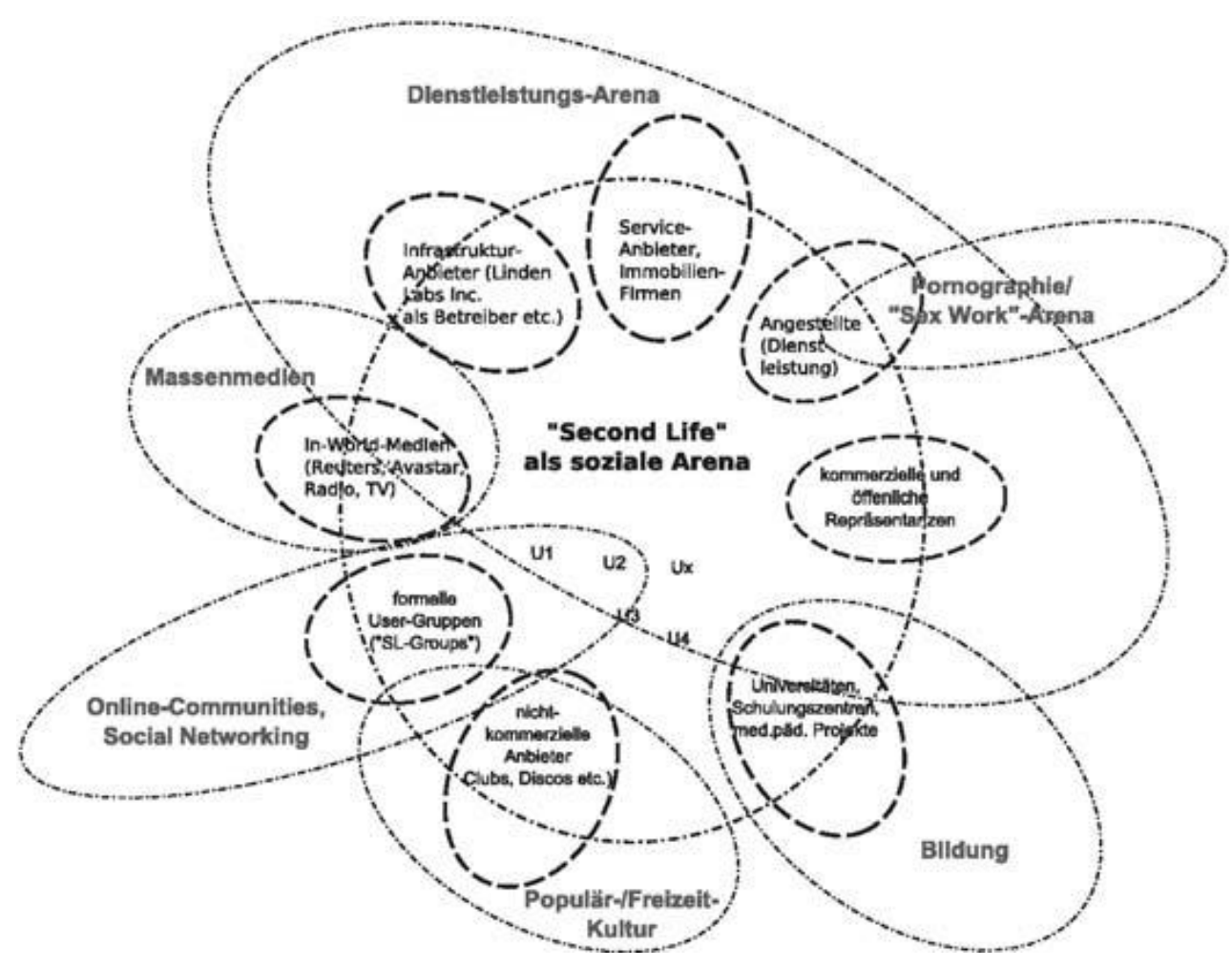

Рис. 1. Карта: «Second Life»: Социальные арены/Миры областей:

Теперь давайте перейдем к подробному обсуждению трех упомянутых структурных 


\section{Структурный уровень I: Виртуальная среда}

Эта структурная область суммирует все, что связано с характером ситуации пользователя в виртуальном мире (с помощью технологии аватара и других аспектов, таких как интерфейс и т.д.), т.е. комнаты, объекты, действия объекта, дизайн и т.д. Виртуальная среда имеет решающее значение для степени сенсорной и социальной живости и даже жизнерадостности цифровой среды, которая, согласно Йоханнесу Фромме (Johannes Fromme, 2008,), является существенным моментом и характеристикой виртуальных миров: чем больше цифровая среда в этом смысле представляет собой живое событие, тем больше, например, возможностей для участия, принятия социальных перспектив и участия в социальном обучении. К виртуальным средам отнсятся следующие аспекты:

1) первым аспектом в смысле базовой структуры обрамления, вслед за Ральфом Шредером (2002b), можно назвать фокусировку цифровой среды. Соответствующий тип социальной сцены, на которой происходят события, будь-то, например, педагогический обучающий и игровой мир, мир дискотек, мир продуктов (Барби, Дисней) и т.д., или открытый, неопределенный виртуальный мир, сопровождается ситуационным кадрированием с точки зрения его пользователей, что в сущности определяет общий характер среды и, таким образом, обрамляет спектр возможных (разрешенных, требуемых) действий. В «теории рамочного анализа» Эрвинга Гоффмана (Goffman, 1996), на которую ссылается Шредер, акцент делается, таким образом, на ансамбле непонятных правил и норм, которые влияют как на действие, так и на восприятие цифровой среды.

2) Техническая и графическая реализация, особенно размерность виртуального пространства, перспектива и базовый репрезентативный характер цифровой среды выделяют наиболее очевидные различия между разными цифровыми средами.

Двухмерные среды являются правилом в аватарных средах 1990-х годов (в основном в виде псевдо-3D визуализаций). Изометрические пространства (так называемые «2.5D миры») в основном распространены в виртуальных мирах для детей и молодежи (например, «Playdo», «Hotel Habbo»), в то время как трехмерные миры являются наиболее требовательной категорией с точки зрения программного космоса и технологий. Аспект перспективы зафиксирован для 2D и $2.5 \mathrm{D}$ миров: каждый из пользователей смотрит на поверхность, или изометрическое пространство с внешней точки зрения; они оторваны от своих аватаров тем, что не двигаются вместе с ними (с лудической точки зрения, эта перспектива очень напоминает кукольные домики; см. рис. 2).

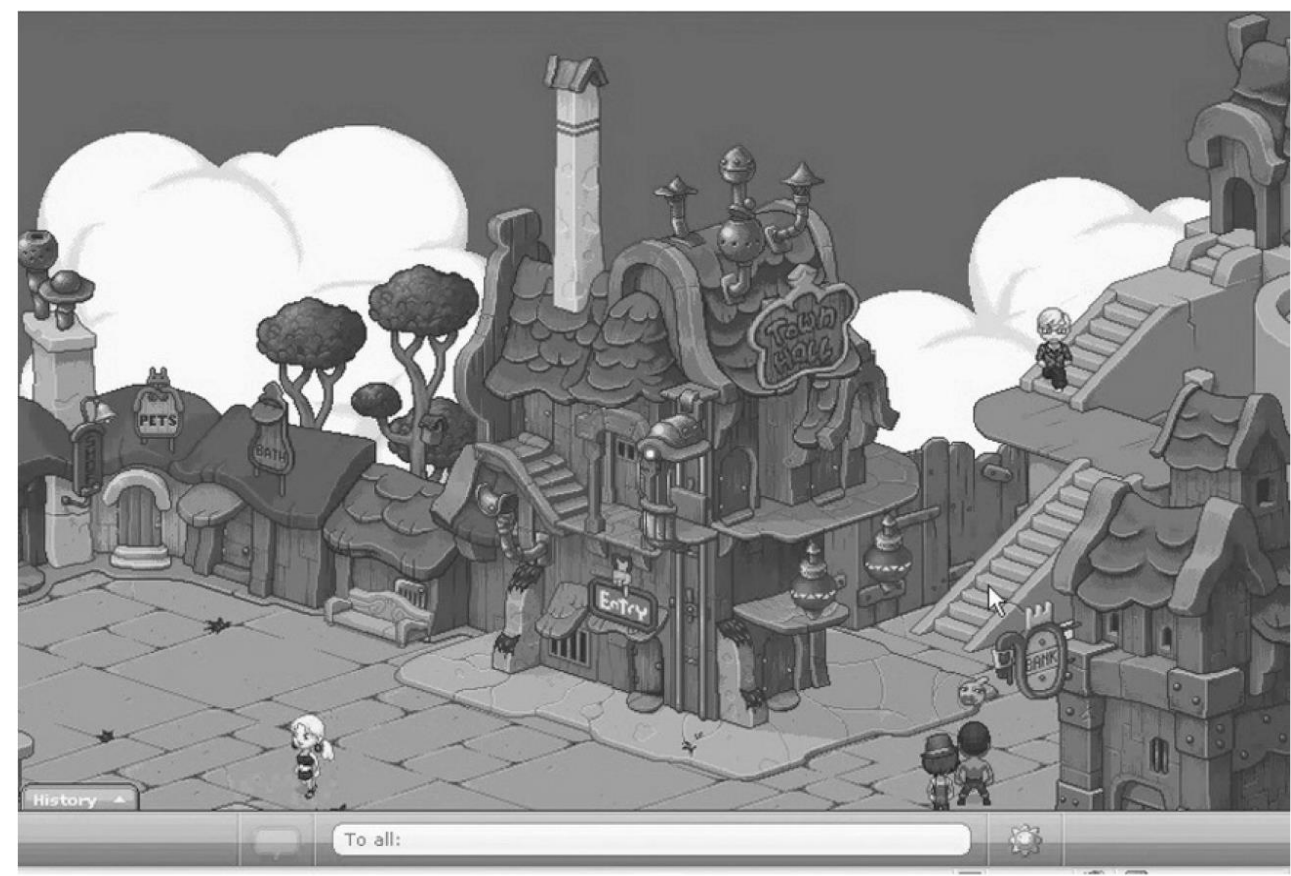

Рис. 2. Виртуальный мир «Плейдо» («Рlaydo»), изометрический или 2.5 D мир. 
В отличие от них, пользователи 3D аватаров обычно ассоциируются с ними, в то время как перспектива эго более распространена в играх (таких, например, как, шутеры от первого лица), в то время как в виртуальных 3D мирах точка зрения зрителя обычно показывает аватар пользователя с немного приподнятой точки зрения сзади, так что аватар находится в поле зрения пользователя во время каждого действия (точка зрения третьего лица). Последний режим создает расстояние до аватара (он визуально обозначен как «другой»), но в то же время позволяет и более четко идентифицировать его действия, которые со-инициализируются и контролируются пользователем.

Наконец, репрезентативный характер цифровой среды можно разделить на две категории: присутствует либо комическая, менее натуралистическая эстетика, либо мир воспроизводит цвет, свет и тень природной среды натуралистическим способом (в рамках технических возможностей). Измерение, перспектива и представительный характер имеют фундаментальное значение для того, каким образом виртуальный мир может быть пережит через его аватары (см. ниже, структура присутствия).

3) Виртуальная среда также включает в себя элементы интерфейса (как аппаратный интерфейс-режимов управления аватаром, так и программный интерфейс, например, графические элементы управления и меню управления). Дизайн интерфейса также имеет большое значение для возможностей опыта в виртуальной среде.

С одной стороны, это интерфейс с физическими действиями пользователя, т.е. прямая связь между физической и цифровой сферами, с другой стороны, дизайн визуального интерфейса определяет видимость среды и, таким образом, избавляет от довольно иммерсивного или довольно дистанционного отношения к событиям в цифровой среде (Fromme, 2006).

\section{Обсуждение теории образования}

Как уже упоминалось выше, образовательная теоретическая значимость виртуальной среды обусловлена соответствующим образом техно-социальной ситуации пользователя. Тема «ситуации онлайн-сообществ» сама по себе относительно сложна, поскольку социальные ситуации всегда кодируются двумя способами: с одной стороны, они возникают из индивидуальных перспектив (или определений ситуации) индивидов, т.е. лежат в эмпирическом измерении; с другой стороны, представляют собой структуру взаимодействия, вытекающую из этих индивидуальных перспектив в целом. Важную роль здесь играют конструктивные элементы: ситуации не даются «объективно» онлайн-сообществу, а являются многоперспективными событиями.

Они представляют собой культурно-исторически сформировавшиеся интерпретации того, что воспринимается как мир (или окружающая среда). Именно в этом и заключается общий потенциал переосмысления и переосмысления ситуаций, который можно считать актуальным с точки зрения теории образования, в смысле изменения мира и самоутверждения. Насколько такая реинтерпретация возможна, зависит не только от ситуационно-интерпретирующего творчества или гибкости участников, но и от ситуационно-недопустимых правил и фрейминга.

Независимо от того, передается она через средства массовой информации, или нет: в социальной среде ситуации всегда учитывают точку зрения других участников ситуации, которая, как правило, известна на низком пороговом уровне восприятия и на основе невербальной коммуникации (например, с помощью соответствующих жестов, движений, мимики, манеры говорить и т.д., служащих ориентирами) и, в случае сомнений необходимо, таким образом, вести переговоры о том, в каких рамках можно совместно понять саму ситуацию.

Здесь структуры власти и признания играют роль, которая даже в повседневной жизни позволяет по-новому интерпретировать ситуации только в определенных рамках. В средах интерактивных медиа добавляются структурные условия медиа, которые направляют спектр возможных ситуаций в социально-техническое русло. Это означает, что, с одной стороны, существуют структурные элементы, которые делают возможным возникновение ситуаций, но, с другой стороны, они имеют более или менее ограничительный эффект.

International Culture \& Technology Studies, Vol. 6, No. 2 
В этом контексте, как минимум, необходимо различать два аспекта в отношении способности виртуальных сред обрамления: 1) чем более неопределенной является «фокусировка», т.е. заданное ситуационное обрамление цифровой среды, тем больше ее потенциал, но при этом также заканчивается и необходимость социальных переговоров о ситуации. 2) Чем в большей степени она присутствует, т.е., чем меньше «погружения» онлайнсообществ в конструкцию интерфейса цифровой среды, тем больше потенциал неявного ситуационного расстояния и, следовательно, ре-экзатив соответствующего медийного окружения (Fromm, 2008; Fromm / Jorissen / Unger, 2008).

\section{Структурный уровень II: Аватары}

Для того чтобы справиться со сложностью технологий аватара, а также для того, чтобы различить области, которые можно функционально или по своим эффектам различать друг от друга, различают три структурные области: а) структура представления (визуальный аспект); b) структура взаимодействия (аспект действия); c) структура присутствия (аспект опыта).

\section{а) Презентационная структура}

Структура презентации относится к визуальному виду аватаров независимо от их интерактивных возможностей (таких как движения и действия). В общем, существует отдельное меню для визуального дизайна, которое представляет аватар в нейтральной среде с небольшим количеством движений тела, или вообще без них. Здесь должно быть сделано различие:

1. Основные формы, которые можно выбрать: спектр варьируется от ограничения на человеческие аватары, гуманоиды (например, фантазийные персонажи, научно-фантастические персонажи) и животных, до совершенно неограниченного выбора базовой формы.

2. Конструктивные параметры: большинство технологий аватара предлагают ряд проектных параметров, но их количество значительно варьируется. Типичными конструктивными параметрами являются, например: пол, форма тела, форма лица, цвет глаз, прическа, цвет волос, одежда и (редко встречающиеся) возрастные характеристики. Иногда есть предложения, которые обеспечивают только базовый выбор готовых аватаров без дополнительных вариантов дизайна.

3. Степени свободы дизайна: влияние параметров дизайна может быть реализовано в очень простом интерфейсе, как дискретный выбор между несколькими альтернативами (например, выбор из 6-8 заданных цветов волос); он также может быть реализован и как Структурная Этнография и Виртуальные Миры. Большинство степеней свободы предлагают такие технологии, которые позволяют создавать собственные поверхности кожи, прически, формы тела, одежды и т.д.

Образовательная значимость презентационной структуры на уровне презентационной структуры аватаров появляется в качестве визуальных артефактов, которые могут иметь высокую артикуляционную ценность. Структура презентации тесно связана с вопросами идентичности и несхожести, «изменения онлайн-сообществ», ролевой игры и маскарада, а также с «микрополитическими» в онлайн-сообществах артикуляциями образа жизни и самоотношений: аватары в социальной цифровой среде, как выразился Мишель Уайт $(2006,123)$, «производят нечто вроде «разговора».

Они отстаивают идеи о том, что такое тела, описывают физиогномику зрителя, представляют то, что ищут зрители, и представляют форму искусства, которую могут прочитать только некоторые зрители. Как уже упоминалось, различные аватарные технологии имеют очень разные степени свободы в дизайне, основных формах и дизайнерских параметрах. Некоторые (немногие) предложения позволяют выбрать только сборный аватар без дополнительных возможностей модификации, что должно выглядеть довольно непродуктивным с точки зрения разрешенного зрительного самовыражения.

Такие предложения, которые конструктивно ограничивают элементы дизайна, доступные для выбора, попадают в аналогичную категорию («MTV Virtual Worlds», например, не позволяют использовать прически противоположного пола, аксессуары и т.д.). Однако, как правило, может существовать и большая свобода (дополнительные элементы), особенно в тех приложениях, 
которые позволяют изначально только ограниченный выбор и логически могут предложить только вполне выборочные спецификации.

Например, типичным для изометрических миров, в основном ориентированных на детей и молодежь (наиболее известные представители — «Hotel Habbo» и «Playdo»), является более или менее дифференцированное меню выбора сборных форм тела, форм и цветов волос, одежды и аксессуаров (солнцезащитные очки и т.д.) [8]. Дополнительные же элементы обычно могут быть приобретены в рамках платного членства. Из технологической структуры следует, что такие предложения влекут за собой приверженность определенным стилям телосложения и представления, которые, однако, могут быть подорваны необычными комбинациями, например, в смысле «эстетики уродливого».

Можно также отказаться от бинарной гендерной матрицы (мужчина / женщина), которая, как правило, реализуется технологически, сочетая соответственно «гендерно-маркированные» элементы (перформативная подрывная деятельность «гетеросексуальной матрицы»), что также может быть реализовано в подобных аватарных приложениях. В целом, однако, эти аватары используются более условно, демонстрируя определенную молодость и субкультурную принадлежность, посредством соответствующего выбора типизирующих причесок и форм одежды.

Возможность денежного приобретения основных форм аватара и аксессуаров также несет в себе аспект отличия. В целом складывается впечатление, что основная артикуляционная логика таких аватарных технологий лежит в большей степени в инсценировке идентичности и молодежных культурных стилей; иными словами, она больше движется в рамках логики идентичности и, в меньшей степени, в рамках логики преобразования самоотношений, что также соответствует фазе психосоциального развития целевой группы таких 2.5D миров, в которых первоочередной задачей является развитие внесемейных групповых связей сверстников.

Третья категория формируется технологиями, позволяющими в значительной степени свободно проектировать компоненты игры, включая аватары. Интересно, что приложения для аватар-чатов 1990-х годов, например, таких как «Virtual Places Chat» (с англ. — «Чат виртуальных мест», прим. пер.) практически не предоставляли возможностей для взаимодействия, а вместо этого предлагали почти полную визуальную свободу дизайна для аватар, так как они состояли только из сменной двухмерной графики, которую пользователи могли загружать в систему. Виртуальный мир «Second Life» же предоставляет одну из самых сложных аватарных технологий: там имеются сотни параметров, с помощью которых можно определить формы тела, глаз, типы кожи (включая старение), цвета и т.д., т.е. они являются практически бесконечно изменяемыми параметрами [9].

Кроме того, любые формы тела могут быть созданы во внешних программах и загружены в систему. Одежда и аксессуары могут быть не только скопированы или куплены «in-world», но также изготовлены или изменены пользователем. Продажа форм тела, поверхностей кожи и одежды составляет значительную часть экономики в «Second Life». Как базовая технология, так и ее конкретная реализация, например, выбор сборных частей, оказывает большое влияние на возможности визуальной артикуляции в смысле выражения культурных, этнических, политических, субкультурных, возрастных, и других аспектов связи. В то время как этническая принадлежность может быть, по крайней мере, показана намёком (с использованием различных цветов кожи), самые лучшие технологии предлагают возможность наносить возрастные метки, такие как морщины или сознательно делать аватар непривлекательным.

Уже на этом этапе культурные или субкультурные формы встречаются со средствами массовой информации. B «Second Life» можно легко увидеть, что существуют очень разные уровни артикуляции и культуры аватар. В то время как значительное количество членов покупают свой аватар (или несколько аватаров) «в мире» в одном из многочисленных магазинов - аватары, так сказать, «с рук», и оснащают их некоторыми аксессуарами (украшениями и т.д.), другие члены идут на гораздо большие усилия, чтобы создать индивидуальные, часто очень необычные аватары из существующих элементов и собственных творений (см.: рис. 3).

International Culture \& Technology Studies, Vol. 6, No. 2 


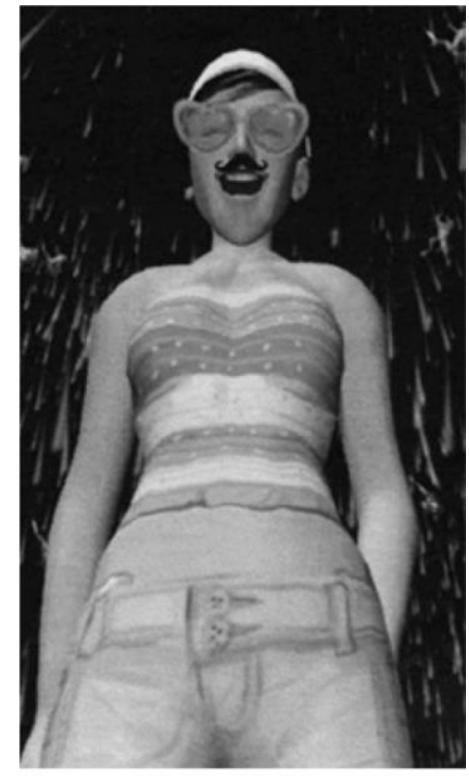

Рис. 3. Гендерные игры в «Second Life» (Quelle: Svarog, 2007).

В этих случаях аватар часто представляет собой явно дискурсивную визуальную форму самовыражения (но не обязательно визуальной саморепрезентации), в которой нормы и идеалы тела, гендерные аспекты, субкультурные и иные исключения обговариваются перформативно (Svarog, 2007).

\section{б) Структура взаимодействия}

Структура взаимодействия является, пожалуй, наиболее сложным структурным аспектом аватаров. В то время как «структура представления» может быть перенесена на другие объекты дизайна (например, на дизайн кукол или марионеток), необычайная «мощь» цифровых объектов в структуре взаимодействия приобретает свою собственную «мощь». Это свойства объектов, которые могут существовать только в цифровой среде - таким образом, мы имеем дело с интерактивным визуальным типом объектов, который является новым и не имеющим прецедента в истории культуры (cp. Bausch / Jörissen, 2005).

Основным, но меняющимся эффектом является то, что действия пользователя взаимодействуют с интерактивными алгоритмами запрограммированных цифровых объектов (например, частей тела аватара). В зависимости от сложности и мощи системы аватар может внести свой вклад во взаимодействия и, таким образом, он становится актером, т.е. независимым, нечеловеческим примером действия (see.: Akrich / Latour 1992, p. 259). В результате получается взаимодействие, «возникающее», в буквальном смысле слова, которое, технически говоря, объединяет как «пользовательский ввод», так и (как таковой) алгоритмические части в амальгаму в конкретном исполнении.

Позы и движения в сложных виртуальных мирах, например, таких как «Second Life», состоят преимущественно из обеих частей. Кроме того, в зависимости от технологии, существует и дополнительное осложнение, связанное с тем, что может быть реализовано автоматическое взаимодействие с другими цифровыми объектами среды (будь-то другие аватары или другие вещи). Дело в том, что результирующие действия аватаров не полностью контролируются ни пользователями, ни алгоритмами, так, что в этом смысле, можно говорить о гибридном актере. На этом структурном уровне необходимо выделить следующее.

\section{Аспекты:}

1. Инициирование и управление действиями: здесь необходимо детально дифференцировать существующие варианты взаимодействия в зависимости от того, управляются ли они пользователем (т.е. «вручную», например, запускаются командой с клавиатуры), как: 
гибридно управляемые (полуавтоматические или опосредованно индуцированные), или алгоритмически индуцированные (полностью автоматические).

2. Варианты вербального взаимодействия: обычно (но не в каждом конкретном случае: см. маленький виртуальный мир «The Endless Forest») [10] вербальная коммуникация является центральным аспектом цифровой многопользовательской среды. Реализуется она либо в текстовом виде, либо голосовым способом («голосовой чат»), выступая, в последнем случае, зачастую, с дополнительной опцией текстового чата.

В текстовом чате, как и в голосовом, необходимо различать, заканчивается ли общение на аватаре, или не на аватаре (например, пузырьками комической речи над головой аватара, или акустической локализацией голоса пользователя на аватаре в виде зависящего от расстояния «голосового чата близости»), или же общение заканчивается отдельно от аватара и, таким образом, не «в мире», а на уровне программного интерфейса (например, подключенные окна чата; внешнее решение голосового чата, такое как в «Skype» [11] или в «Teamspeak» [12]. Этот аспект вербального взаимодействия особенно важен для структуры присутствия (см. ниже).

3. Варианты невербального взаимодействия: к ним относятся неявные и эксплицитные жесты (позы, позы, движение глаз, мимика; размахивание, хлопки, жесты руками и т.д.), двигательные жесты (такие как ходьба, танцы, бег, плавание, полет), а также варианты пространственных движений (например, изменение положения аватара в пространстве изображения, позволяющее аватару приближаться или отдаляться от других аватаров, участие в неформальных или формальных «сценических аранжировках», таких как формирование пространственных групп; обычные «спортивные» режимы движения, такие как автогонки и т.д.).

Здесь необходимо учитывать, могут ли аватары сталкиваться с другими объектами и аватарами, или же программирование не предусматривает столкновений. Образовательная значимость структуры взаимодействия, как следует из названия, в первую очередь связана с символическими вариантами действий в виртуальных средах. На этом уровне можно рассмотреть как предварительный план действий [10] (http://tale-of-tales.com/ The Endless Forest fe (as-if action)), с одной стороны, но также и повторные «переселения», которые являются результатом переноса повседневных моделей и норм действий в новый контекст (контекст виртуальных миров). С другой стороны, речь идет о потенциальном восстановлении субъекта действия на основе гибридных техносоциальных структур действия.

Таким образом, уровень «структуры взаимодействия» фокусируется на особой характеристике многих аватарных технологий, а именно - их социально-динамичном, частично самодостаточном характере. Вопрос здесь в том, кто осуществляет действия в цифровой среде, основанной на аватаре, и на каком уровне они осуществляются, т.е. на какой социальный мир символически ссылаются действия, осуществленные с аватаром. Для некоторых видов действий или коммуникативных актов может быть достаточно ясно показано, что это действия пользователя аватара: если, например, в «Second Life» «реально экономический» процесс покупки инициируется щелчком по объекту, и аватар в этом присутствует, но вряд ли при этом можно утверждать, что сам аватар что-то «покупает». Аналогично, если аватар делает оскорбительные жесты по отношению к другим, вряд-ли можно сказать, что один аватар оскорбляет другого (хотя уже неясно, обращается ли действующий пользователь в таком случае к другому пользователю или к другому аватару своим оскорбительным жестом).

Однако во многих других случаях ситуация менее ясна. Если танцевальное движение активируется нажатием клавиши, то сложно сказать, что пользователь танцует. Нажатие на клавишу компьютерной клавиатуры является аналитическим, т.е. техническим, но при этом также четко оно отделено от самого виртуальных движений аватара. Аватар танцует? Или точнее, можно ли поставить вопрос о том: выполняет ли он виртуальные движения в виртуальном пространстве, которые структурно сопоставимы с танцевальными движениями? И если пользователь меняет танцевальные шаги одним нажатием кнопки в соответствии с изменением музыки в виртуальной дискотеке и движениями других аватаров, то кто тогда танцует?

Пример впечатляюще показывает: в конце концов, уже невозможно различить, какая часть в ансамбле пользователя и аватара представляет собой конкретный пример действия. Варианты

International Culture \& Technology Studies, Vol. 6, No. 2 
взаимодействия на аватаре - взгляд, жесты, мимика, позы, жесты движения (ходьба, бег, плавание и т.д.) и связанные с ними пространственные (непрерывные) возможности движения относятся к антропологически очень сложному вопросу о теле, его пределах и статусе как объекта социально-культурного строительства.

Тот факт, что жесты и формы движения потенциально имеют артикулятивный смысл, уже очевиден из того факта, что жесты аватаров спроектированы и интерпретированы по-разному в зависимости от культуры (Koda, 2007; в целом о построении «расы» с аватарами: Nakamura, 2007, p. 131). Поскольку невербальная коммуникация играет важную роль во всех культурах, эта информация поначалу может вызывать лишь ограниченное удивление. Жесты аватара представляют собой здесь скорее культурные перформансы, которые приобретают ярко выраженный перформативный характер именно благодаря «удвоению», т.е. ссылке на культурные жесты происхождения.

Это особенно верно для субкультурных жестов и поз, которые указывают на принадлежности и разграничения. Особый миметический потенциал есть и в том, что культурно «крутые» позы молодых людей и часто очень «искусные» движения аватаров, безусловно, могут показаться достойными подражания, например, упомянутые выше танцевальные движения в виртуальных «диско-мирах» [13], специализирующихся на них. Если технологии аватары позволяют программировать или модифицировать сами жесты и движения, что, однако, является скорее исключением, то они могут стать особенно сложным и дифференцированным (суб)культурным артикуляционным средством. В настоящее время «Second Life» является наиболее технологически продвинутой платформой и в этой области.

Сценарии для жестов можно запрограммировать и поместить в виртуальный мир в любой желаемой форме, как правило, в виде маленьких шариков (если на них кликнуть, то соответствующий жест выполняет собственный аватар). Существующие стандартные жесты в основном различают женские и мужские жесты (в том смысле, что жестовые шарики окрашены в розовый или синий цвет) [14]; это техно-социальная гендерная деятельность («Doing Gender»), основанная на гетеросексуальной матрице, хотя и адаптирована, но также может иметь как умеренный, так и подрывной характер, например, когда гей-сообщества во «Второй Жизни» программируют свои собственные (светло- и темно-синие) шарики.

Таким образом, жесты и движения аватаров играют ключевую роль для вида социальной общности, культивируемой в цифровой среде. В силу своих социально-технологических структурных характеристик, аватары являются особенно сложным примером того, что в теории актерских сетей (АНТ) называют «актёром». Акрич и Латур понимают их как нечеловеческие элементы социальных действий и цепочек действий. Актером является «любое действие или перемещение действия; само действие определяется списком представлений в ходе испытаний; из этих представлений вычитается набор компетенций, которыми наделен актер [14]»; в то время как актером является «актер, наделенный персонажем (обычно антропоморфным)» (Akrich / Latour 1992, p. 259). В этом общем определении «неразумные» акты могут быть также тематизированы как действующие лица [15].

Аватары, таким образом, не функционируют как пассивные объекты (http://www.vside.com, http://tinyurl.com/Second-Life-Gendergesten) [15]. С точки зрения АНТ, аватар находится в «определенной» цепочке человеческих и нечеловеческих актеров - пользователей, экранов, компьютерных мышей, виртуальных объектов, графических карт, подключений к Интернету, клавиатур, программ (софта) и т.д., которые формируют тип создаваемых подключений.

Таким образом аватар, в смысле таких актерских сетей (АНТ), на наш взгляд, мог бы представлять собой особо сложный социальный объект, поскольку он расположен на пересечении нескольких линий электропередач: технической линии (например входы интерфейса - связь с сервером - цифровая среда / виртуальный мир), визуальный (взгляд - экран изображения изображение), социальный (пользователь — аватар — аватар — пользователь) и тактильный (рука - компьютерная мышь - движения / ярлыки аватара). В связи с актерским характером аватаров, взаимоотношения пользователя-аватара не могут быть поняты в соответствии с субъектнообъектной схемиотикой, например, моделью кукольного игрока. Социальная динамика, создаваемая аватарным взаимодействием, оказывает влияние на пользователя.

Культура и технологии, Том 6, № 2 
Подобная ретроактивная связь была исследована Ником Йи в социально-психологической среде и была названа «эффектом Протеуса» (Yеe, 2007). В серии экспериментов профессиональным группам был присвоен аватар, который соответствовал различным значениям привлекательности (например, в отношении размера тела и формы лица). Так, в виртуальной комнате испытуемые видели свой собственный аватар, отраженный в (виртуальной) стене. Другой участник, присутствовавший в виртуальной комнате, затем общался с этим аватаром, или испытуемым, но не видел того же аватара, что и испытуемый, но при этом аватаром всегда оставался один и тот же (нейтрально привлекательный) виртуальный человек.

В результате Йи смог продемонстрировать весьма существенные изменения в поведении испытуемых: оснащенные привлекательными аватарами, держались на значительно меньшей межличностной дистанции от других аватаров, предоставляли значительно больше информации о себе и были склонны использовать преимущество своих оппонентов в игровых настройках. Йи заключает: «появление наших аватаров формирует то, как мы взаимодействуем с другими. По мере того, как мы выбираем самопредставление в виртуальной среде, наше самопредставление, в свою очередь, формирует наше поведение. Эти изменения происходят не в течение нескольких часов или недель, а в течение нескольких минут» (Yеe, 2007). Такие атрибуты Йи относятся к визуальной самозависимости (т.е. в нашей структурной дифференциации они скорее должны быть отнесены к структуре презентации).

Марк Хансен (2006) философствует о гораздо более глубоком физическом моменте, который влияет на структуру взаимодействия. Хансен видит виртуальную телесность, фундаментально характеризующуюся тем, что техногенный третий элемент вписывает себя во взаимоотношения между телесной схемой и образом тела, т.е. между тактильно-моторным пространством переживания тела и зрительно-проприемлемым самоощущением. В этом отношении виртуальное тело не следует понимать как информационную или цифровую абстракцию повседневного тела. Скорее, мы должны говорить о «органе, которому она подчинена, и который был создан в результате неизбежной и расширяющей возможности технической экстерриториализации» (Hansen, 2006, 20). Хансен называет такой орган, который может быть реализован только через связь с технологией, «телом в коде» и приписывает этому созвездию потенциал для того, чтобы отделить схему тела, т.е. измерение тактильного опыта, от образа тела с целью «увеличения его агентуры как воплощенного существа» (Hansen, 2006, 20).

По мнению Хансена, посредничество через визуальное техно-тело возможно при определенных условиях и развивает свою аргументацию на основе интерактивных произведений Моники Флейшман и Вольфа Штрауса, т.е. в высокоразвитой и переэкстрагированной художественно-экспериментальной среде, которая имеет потенциал для нового открытия тела. Феноменологическая теоретизация виртуальной телесности Хансена соответствует результатам исследований аватара в том, что, как указывает Ральф Шредер (Ralph Schroeder, 2002b), ощущение физического присутствия в виртуальном месте, «ощущение бытия там», не зависит от исходного натуралистического объекта. Скорее, именно интерактивность и реактивность аватаров, а также их способность общаться не в устной форме, являются причиной этого.

В этом смысле Том Бёллсторф, проводивший многочисленные интервью во время трехлетнего наблюдения за участниками «Second Life», сообщает о важности агентства, связанного с аватарами «Second Life» и отметил, что «экспериментирование с внешним видом или поведением в «Second Life» потенциально открывает новые возможности для мышления о вещах в реальной жизни. «Несмотря ни на что, кто я такой, кажется, все еще выходит наружу, так что, возможно, я обнаруживаю свою сущностную природу [через мой аватар]; заметив, что «мое слабое «я» становится больше похожим на мой личностный аватар», как будто SL [Second Life] выросла на мне и зациклилась» (Boellstorf, 2008, s.148).

Эта тема «Второй Жизни», позволяющая получить доступ к внутреннему «Я», которое в реальном мире маскируется неизбирательным воплощением и социальными обязательствами, была «общей», утверждает Беллсторф (Boellstorf, 2008, 148), и делает вывод из своих наблюдений: «аватары были не просто плацдармами для самосознания, но и местами самосознания» (Boellstorf, 2008, s. 149).

International Culture \& Technology Studies, Vol. 6, No. 2 
Упомянутые здесь исследования с разных дисциплинарных точек зрения дают понять следующее: чем выше наличие аватарной технологии (как бы она ни была реализована) в смысле такого агентства, тем больше вероятность того, что это агентство откроет новые пространства для самопознания. Подобное присутствие соответствует социальному сосуществованию в цифровой многопользовательской среде, т.е. возможности переживания «артикулированного другого». Только благодаря этому зрительные и жестикуляционные артикуляции в конечном итоге становятся социально эффективными в делиберативном цифровом пространстве.

Резюме: Об образовательной значимости аватар

Каждая технология аватара имеет характеристики, отличные от других. Выделенные здесь структурные уровни показывают потенциал виртуальной телесной реальности, который актуален как с точки зрения антропологии средств информации, так и с точки зрения теории образования, и который, учитывая нынешние тенденции, может оказаться в центре (заинтересованного или приверженного) интереса в будущем. На основании вышеизложенных наблюдений представляется уместным сказать, что «агентство» в виртуальных мирах — это гибрид пользователя / аватара, т.е. гибридный актор.

Взаимосвязь пользователя и аватара, в конечном счете, очевидна на всех структурных уровнях анализа: во-первых, на уровне виртуальной среды (структурный уровень I) в цифровой техносоциальной среде (например, виртуальный мир). Во-вторых, на уровне структуры презентации происходит наложение собственных и медиально предструктурированных артикуляционных вариантов. В-третьих, на уровне структуры взаимодействия можно наблюдать гибридизацию (целевую) действий пользователя и полуавтономных действий аватара. И наконец, в-четвертых, на уровне структуры присутствия можно наблюдать гибридизацию опыта в том смысле, что пользователь вступает в тесную идентификационную связь со своим аватаром («Эффект Протеуса»), а за ее пределами границы собственной физичности растворяется в визуально-моторном измерении опыта.

\section{Структурный уровень III: Общественные функции}

В качестве третьего структурного уровня следует рассматривать общественные аспекты виртуальных миров, которые в значительной степени совпадают с теми, что представлены в онлайн-этнографии Винфрида Мароцкого (Marotzki, 2003). Существуют типичные перекрытия с другими структурными уровнями; например, в отношении коммуникационных функций аватаров и публичного, или частного характера пространств в виртуальных средах. Мароцкий (2003) выделяет семь онлайн-этнографических структурных признаков:

1) Определяющая метафора: На ранней стадии развития Всемирной паутины (WWW) представление онлайновых сообществ, основанных на WWW, как правило, следовало определенным метафорам, которые в то же время символизировали структуру навигации, а также возможности взаимодействия и коммуникации (например, «киберкафе» для онлайнового чата). Таким образом, этот аспект общинной этнографии может быть надлежащим образом рассмотрен в Виртуальном мире под названием «Виртуальная среда».

2) Каждое онлайн-сообщество имеет свою специфическую социографическую структуру. Сюда входят условия и процедуры доступа (регистрация), набор правил, система статуса (неоплачиваемые, простые и премиальные членства можно найти почти во всех коммерчески эксплуатируемых сообществах), система вознаграждения и санкций, система «приятель и игнор» (идентификация друзей/врагов), а также часто очень важные (обычно свободно конфигурируемые) группы.

Эти характеристики могут иметь решающее значение для формы установившихся социальных отношений в онлайн-сообществах или виртуальных мирах. Тип доступа, например (анонимная регистрация против регистрации с полным именем и проверкой адреса электронной почты, возможно, даже проверкой личности), определяет, является ли персонаж сообщества скорее необязательным или принимает форму «серьезной игры», в которой участники, в конечном счете, взаимодействуют со своей реальной идентичностью. 
Система денежных подарков и санкций способствует определенным (предполагаемым) регулярным формам действий в общинах, таким, как участие в дискуссиях на форумах, которые часто имеют ритуальный и общинный характер. Наконец, система «приятель» создает структуры признания и, таким образом, может создавать стабильные сообщества внутри сообщества (в зависимости от реализации и использования), а также преобразовывать статус, например, включать в существующую группу равных. Наконец, группы предлагают вариант организации сообщества, как правило, исходя из интересов.

«Second Life» является в этом плане хорошим примером: здесь групповая функция в первую очередь размещена в отдельном интерфейсе опций, который не принадлежит самому миру. Членство в группе может по желанию отображаться «в мире» в виде соответствующих ярлыков аватара (имя и название группы затем отображаются над заголовком), так, что членство в группе может быть доминирующим признаком.

3) Онлайн-сообщества зависят от участия их членов. Степень совместного определения регулируется структурой участия, которая дифференцированно определяет варианты совместного определения членов. Она сильно различается в разных общинах и часто зависит от статуса членов (новички имеют меньше прав, чем давние активные члены, платят больше, чем неоплачиваемые члены и т.д.).

Можно проводить различие между участием с точки зрения содержания (например, действия в мире), структурным участием (например, такое изменение мира как строительство) и организационным участием (например, - совместное принятие решений об основном характере и фундаментальных изменениях виртуального мира). Эти виды участия потенциально сопровождаются различной степенью вовлеченности и приверженности.

4) Каждое онлайн-сообщество имеет, по крайней мере, один способ общения между своими членами. Как правило, это целый набор различных вариантов, составляющих специфическую коммуникационную структуру сообщества. Для веб-сообществ это включает в себя как «индивидуальные», так и «много-много-много» коммуникационные услуги (чат, приватный чат, форумы, электронная почта, обмен мгновенными сообщениями, SMS-интерфейс, списки рассылки и т.д.).

Что касается виртуальных миров, то структура коммуникации в значительной степени реализуется через структуру взаимодействия аватарной технологии, но существуют и дополнительные каналы коммуникации, которые могут быть расположены внутри интерфейса виртуального мира, и при этом не принадлежат к тому, что происходит в мире (соответственно, это также включает в себя коммуникационные возможности на дополнительно подключенных веб-сайтах).

5) Сообщества ориентированы на более или менее четко определенные общие темы или интересы. Под информационной структурой должны пониматься все услуги, на основе которых членам предоставляется информация, имеющая к ним отношение. Это может быть тематическая или общественная информация, например, информационные бюллетени, тематические сборники ссылок и базы данных, календари для организации совместных мероприятий, а также глоссарии и вики-сайты, интегрированные в веб-сайт.

Этот аспект очень важен для виртуальных миров, так как с ними, как правило, нелегко работать. Поэтому очень часто новички сначала знакомятся с виртуальной средой с помощью обучающих видеороликов или даже делают первые шаги в самой виртуальной среде (в «Second Life», например, новички автоматически приземляются на «Островах Ориентации»).

6) Структура представления сообщества включает в себя управление идентификацией, доступное во всех онлайн-сообществах, т.е. доступные варианты самопредставления отдельных членов. Кроме того, некоторые сообщества предлагают возможность проведения презентации на групповом уровне или даже визуализации сетей друзей.

Презентация может проходить в веб-сообществах на частных или полугосударственных аренах (собственная домашняя страница или веб-блог внутри страниц сообщества; как удостоверение личности с перечисленными личностными характеристиками и предпочтениями). В

International Culture \& Technology Studies, Vol. 6, No. 2 
случае виртуальных миров, аватар, конечно, является центральным примером структуры презентации, в то время как зачастую дополнительные общественные и частные арены (рroстраницы) присутствуют вне виртуальной среды.

7) Наконец, взаимосвязь между онлайновым и оффлайновым режимами часто играет важную роль: многие сообщества имеют структурные механизмы, которые позволяют их членам встречаться друг с другом на регулярной основе или которые конкретно содействуют проведению таких встреч, например, направляя даты встреч или других мероприятий заинтересованным сторонам по электронной почте. Это могут быть пользовательские встречи тематических сообществ или встречи между отдельными членами или группами.

Кроме того, отношения «online-ofline» включают в себя также интегрированные в общины предложения магазинов, которые приводят, например, к реальным поставкам товаров. При этом в большинстве виртуальных миров существуют дорогостоящие предложения магазинов, предлагающих аксессуары и одежду для аватара или мебель и предметы для обустройства собственной виртуальной квартиры. Особым случаем уже упоминались такие миры, как «Second Life» с его гибридной экономикой и, соответственно, разнообразием внутрисетевых соединений.

\section{Заключение}

Предыдущие замечания были направлены на то, чтобы показать структурную сложность виртуальных миров, ввести возможные категории анализа и очертить возможные связи в теории образования. Выделенные здесь структурные уровни показывают потенциал виртуальных миров и аватарных технологий, которые актуальны как с медиа-педагогической, так и с образовательнотеоретической точки зрения, и которые, с учетом нынешних тенденций, возможно, в будущем будут все больше и больше находиться в центре общественного внимания. Каждый виртуальный мир обладает характеристиками, которые отличаются от других, часто в существенных аспектах.

Вслед за Норбертом Медером, образовательная значимость цифровой среды может быть охарактеризована тем, что она соответствует «логике сокращения до существенного»: с «одной стороны, пространство для образовательных процессов сужается, с другой стороны, открывается неопределенное пространство, в котором возможно «что угодно». Это означает, что «свободное пространство предоставляется творчеству и воображению» (Meder, 2008, s. 231), где их потенциал заключается как в формативной артикуляции, так и в актерском участии, но это происходит, вопервых, в разной степени, а во-вторых, в разной ориентации:

1) Как видно из различных структурных аспектов, отношения между узостью и свободой в различных виртуальных мирах очень различны. Существуют также примеры, которые оставляют мало места для личного самовыражения и вместо этого предлагают лишь стереотипные предложения в отношении идентичности и взаимодействия. Другие миры успешно пытаются найти баланс между «дидактическим сокращением» и свободой дизайна и действия, предлагая практически неограниченные возможности, но требуют при этом выраженного желания учиться.

2) Кроме того, виртуальные миры предлагают очень разные фреймы, что также должно быть принято во внимание. Базовый характер мира может быть в большей степени ориентирован на педагогику, знание или игру; он может сосредоточиться на предложениях идентичности, заставляя отождествлять себя с молодежными культурными субкультурами и сценами, или он может дать возможность играть с идентичностью, пробуя другие формы, которые подрывают границы (киборги, мульти- или асексуальные существа, химеры и т.д.). Наконец, этот базовый характер может быть сосредоточен на потенциалах действий (например, там, где на первый план выходят общие формы действий и/или участия в построении мира, или даже на реальных социальных аренах, оказывающих влияние на виртуальный мир).

В целом, становится ясно, что виртуальные миры представляют собой медиа-сферу с разнообразным учебным и образовательным потенциалом. Они дают возможность ориентировочно пережить и расширить себя и мир в альтернативном пространстве. Однако, учитывая описанные выше сложности, об этом потенциале ни в коем случае нельзя судить в общих чертах. Скорее, для каждого конкретного случая требуется индивидуальный или сравнительный анализ структурных аспектов, имеющих отношение к образованию, и их взаимодействия. 


\section{Сноски}

1. Различие между Виртуальными мирами и настоящими Виртуальными игровыми мирами может быть определено формальными особенностями игры, такими как наличие явных или подразумеваемых игровых целей и правил. Виртуальные миры не имеют предопределенной цели действия, или повествовательной последовательности, лудической топологии в смысле мест, функциональных для определенных аспектов игры и т.д. У них также нет правил игры, но, как и у любого онлайн-сообщества, у них есть социальные правила поведения. Виртуальные миры вполне могут содержать разнообразные встраиваемые игры. Однако, это не делает их в игру больше, чем флэш-игры в веб-браузере сделает игру из всемирной паутины или социальной сети, в которую они интегрированы.

2. Некоторые примеры видеоматериалов собраны по адресу http://www.youtube.com/user/joeriben; (выборочный) список виртуальных миров можно найти по адресу http://www.diigo.com/list/benjamin/virtualworlds.

3. Например, платформа «Активные миры» («Active Worlds»), которая до сих пор широко используется, существует с 1995 г. Особенно в виде серых чат-приложений, таких как «Дворец» («Тhe Palace»), или серых МУДов, таких как ««Космическая станция Чата Миров» (Worlds Chat Space Station»), такие простые виртуальные миры становятся все более популярными с середины 1990-х гг. К концу этого десятилетия все чаще появляются трехмерные миры в стиле «Активных миров». «Мув Роуз 3D» («Moove Rose 3D», 1999) и псевдо 3D миры «Отель Хаббо» («Habbo-Hotel», 2000) и «Плейдо» (« Playdo», 2000) являются одними из самых распространенных таких ранних предложений.

4. Поскольку этот подход был первоначально разработан с использованием аватарных технологий, некоторые отрывки из следующего текста уже были опубликованы в других местах (см., в частности, Jörissen / Marotzki, 2009, p. 224 и далее).

5. Boellstorf (2008); см. также Журнальные научно-популярноные, но, те мне менее, интересные полевые отчеты, интересные полевые репортажи Guest (2007) и Stöcker (2007).

6. В простой форме, а именно со статичными «аватарами» (не с интерактивными аватарами, как здесь обсуждалось, a c маленькими квадратными изображениями). Gravatar.com - это широко распространенный сервис, позволяющий динамически интегрировать собственный «аватар» в разных местах WWW (например, рядом с вашими собственными комментариями в зарубежных блогах и т.д.).

7. http://tinyurl.com/Weblin-Demo

8. http://tinyurl.com/Playdo-Avatar-Creation-Demo

9. http://tinyurl.com/Second-Life-Avatar-Generierung

10. http://tale-of-tales.com/TheEndlessForest

11. http://www.skype.com

12. http://www.teamspeak.com

13. http://www.vside.com

14. http://tinyurl.com/Second-Life-Gendergesten

15. С точки зрения АНТ, аватар находится в «определенной» цепочке человеческих и нечеловеческих актеров - пользователей, экранов, компьютерных мышей, виртуальных объектов, графических карт, интернет-соединений, клавиатур, программ (софта) и т.д., каждый из которых формирует тип создаваемых связей. Входы интерфейса - связь с сервером - цифровая среда/виртуальный мир), визуальный (взгляд - экран изображения — изображение), социальный (пользователь — аватар аватар - пользователь) и тактильный (рука — компьютерная мышь - движения/ярлыки аватара). Аватар, таким образом, является объектом, где эти цепочки рук в смысле АНТ перекрываются и трансформируются, оказывая влияние друг на друга.

16. http://www.vpchat.com/

17. http://tinyurl.com/virtual-eve

\section{References}

[1] Akrich Madeline/Latour, Bruno (1992): A Summary of a Convenient Vocabulary for the Semiotics of Human and Nonhuman Assemblies. In: Bijker/Law 1992, p. 259-264.

[2] Bachmair, Ben/Diepold, Peter/de Witt, Claudia (Hrsg.) (2003): Jahrbuch Medienpädagogik 3. Opladen: VSVerlag.

[3] Bailenson Jemery N./Beall, Andrew C. (2005): Transformed Social Interaction: Exploring the Digital Plasticity of Avatars. Online: http://vhil.stanford.edu/pubs/2006/bailenson-digital-plasticity.pdf [7.6.2009].

[4] Barnes, Brooks (2007): Web Playgrounds of the Very Young. In: New York Times vom 31.12.2007. Online:

[5] http://www.nytimes.com/2007/12/31/business/31 virtual.html [7.6.2009]

[6] Bausch, Constanze/Jörissen, Benjamin (2005): Das Spiel mit dem Bild. Zur Ikonologie von ActionComputerspielen. In: Wulf/Zirfas 2005, s. 345-364.

International Culture \& Technology Studies, Vol. 6, No. 2 
[7] Becker Barbara/Mark, Gloria (2002): Social Conventions in Computer-mediated Communication: A Comparison of Three Online Shared Virtual Environments. In: Schroder 2002a, 19-39.

[8] Bente Gary/Krämer, Nicole C./Petersen, Anita (Hrsg.) (2002): Virtuelle Realitäten. Göttingen: Hogrefe.

[9] Bijker Wiebe E./Law, John (Hrsg.) (1992): Shaping Technology / Building Society: Studies in Sociotechnical Change. Cambridge, Mass.: MIT Press.

[10]Boellstorf, Tom (2008): Coming of Age in Second Life: An Anthropologist Explores the Virtually Human.Princeton: Princeton University Press.

[11]Bormann,Sven (1994): Virtuelle Realität: Genese und Evaluation. München: Addison Wesley.

[12] Butler Judith (1991): Das Unbehagen der Geschlechter. Frankfurt am Main: Suhrkamp.

[13] Clarke Adele (2005): Situational Analysis. Grounded Theory after the Postmodern Turn. Thousand Oakes:Sage.

[14] Svarog, Forseti (Hrsg.) (2007): Avatar Expression. (Selbstverlag).

[15] Dery Mark (1996): Escape velocity. Cyberculture at the end of the century. London: Grove Press. Featherstone, Mike/Burrows, Roger (Hrsg.) (1995): Cyberspace, Cyberbodies, Cyberpunk. London: Sage.

[16]Ferdig Rick (Hrsg.) (2008): Handbook of Research on Effective Electronic Gaming in Education. Hershey: Information Science Reference.

[17] Freyermuth, Gundolf S. (1996): Cyberland. Eine Führung durch den High-Tech-Underground. Berlin: Rowohlt.

[18] Fromme Johannes (2006): Socialisation in the Age of New Media. In: MedienPädagogik 11/2006. Online:http://www.medienpaed.com/05-1/fromme05-1.pdf [16.7.2007].

[19] Fromme, Johannes (2008): Virtuelle Welten und Cyberspace. In: Gross/Marotzki/Sander (2008), p. 169-201.

[20] Fromme Johannes/Jörissen, Benjamin/Unger, Alexander (2008): (Self-) Educational effects of computer gaming cultures. In Ferdig 2008, p. 757-775.

[21] Goffman Erving (1996): Rahmen-Analyse. Ein Versuch über die Organisation von Alltagserfahrungen. (4. Au ). Frankfurt am Main: Suhrkamp.

[22] Gross Friederike von/Marotzki, Winfried/Sander, Uwe (Hrsg.) (2008): Internet - Bildung Gemeinschaft.Wiesbaden: VS Verlag.

[23] Hansen, Mark B. N. (2006): Bodies in code. Interfaces with digital media. New York: Routledge.

[24] Hillis Ken (1999): Digital sensations: space, identity, and embodiment in virtual reality. Minnesota: University of Minnesota Press.

[25] Ishida Toru/Fussell, Susanne R./Vossen, Piek T. J. M. (Hrsg.) )(2007): Intercultural Collaboration (IWIC 2007). Wien/New York: Springer.

[26] Jensen, Jens F. (2001): Virtual Inhabited 3D Worlds: Interactivity and Interaction Between Avatars, Autonomous Agents and Users. In: Qvortrup 2001, p. 23-47.

[27] Jörissen Benjamin (2008): The Body is the Message. Avatare als visuelle Artikulationen, soziale Aktanten und hybride Akteure. In: Paragrana 17/2008, p. 277-295.

[28] Jörissen, Benjamin/Marotzki, Winfried (2009): Medienbildung — eine Einführung. Theorie — Methoden — Analysen. Bad Heilbrunn: Klinkhardt/UTB.

[29] Koda Tomoko (2007): Cross-Cultural Study of Avatars Facial Expressions and Design Considerations Within Asian Countries. In: Ishida/FusselI/Vossen 2007, p. 207-220.

[30]KZERO (2009): Virtual Worlds Registered Accounts Q1 2009. Online: http://www.kzero.co.uk/blog/?page_id=2537 [7.6.2009].

[31]Latour Bruno (2007): Reassembling the social. an introduction to Actor-Network-Theory. Oxford: Oxford University Press.

[32] Marotzki Winfried (1990): Entwurf einer strukturalen Bildungstheorie. Biographietheoretische Auslegungvon Bildungsprozessen in hochkomplexen Gesellschaften. Weinheim: Deutscher Studienverlag.

[33] Marotzki Winfried (2003): Online-Ethnographie - Wege und Ergebnisse zur Forschung im KulturraumInternet. In: Bachmair/Diepold/de Witt 2003, s. 149-165. Online: http://www.unimagdeburg.de/iew/web/Marotzki/03/virt_Communities/Marotzki_2003.pdf [20.06.2009].

[34] Marotzki Winfried (2008): Weisen der Artikulation in bildungstheoretischer Perspektive am Beispiel von Web 2.0 Phänomenen. In: Schachtner/Höber 2008, s. 57-70.

[35] Meder Norbert (2008): Bildung und virtuelle Welten-Cyberbildung. In: Gross/Marotzki/Sander 2008, p. 227239.

[36] Nakamura Lisa (2007): Digitizing Race: Visual Cultures of the Internet. Minnesota: University Of MinnesotaPress.

[37]Petersen Anita/Bente, Gary/Krämer, Nicole C. (2002): Virtuelle Stellvertreter: Analyse avatar-vermittelter Kommunikationsprozesse. In: Bente/Krämer/Petersen 2002, 227 - 253.

[38] Qvortrup Lars (Hrsg.) (2001): Virtual Interaction: Interaction in Virtual Inhabited 3D Worlds. London: Springer.

[39] Rheingold Howard (1992): Virtuelle Welten. Reisen im Cyberspace. Reinbek: Rowohlt.

[40] Sandbothe Mike (2001): Pragmatische Medienphilosophie: Grundlegung einer neuen Disziplin im Zeitalter des Internet. Weilerswist: Velbrück Wissenschaft.

[41] Schachtner Christina/Höber, Angelika (Hrsg.) (2008): Learning Communities. Das Internet als neuer Lernund Wissensraum. Frankfurt am Main: Campus. 
[42] Schlette Magnus/Jung, Matthias (Hrsg.) (2005): Anthropologie der Artikulation. Begrif iche Grundlagen und transdisziplinäre Perspektiven. Würzburg: Königshausen \& Neumann.

[43] Schroeder Ralph (Hrsg.) (2002a): The social life of Avatars. presence and interaction in shared virtual environments. London: Springer.

[44] Schroeder Ralph (2002b): Social Interaction in Virtual Environments: Key Issues, Common Themes and a Framework for Research. In: Schroeder 2002a, p. 1-18.

[45] Schroeder Ralph/Axelsson, Ann-So e (Hrsg.) (2006): Avatars at Work and Play. Collaboration and Interaction in Shared Virtual Environments. Dordrecht: Springer.

[46] Stephenson Neal (1993): Snow crash. London: Bantam.

[47] Steurer Siegfried (1996): Schöne neue Wirklichkeiten? Die Herausforderung der virtuellen Realität. Wien:Wuv Universitaets Verlag.

[48] Taylor,T. L. (2002): Living Digitally: Embodiment in Virtual Worlds. In: Schroeder 2002a, 40-62.

[49] White,Michele (2006): The body and the screen. theories of internet spectatorship. Cambridge, Mass: MIT Press.

[50] Wulf Christoph/Zirfas, Jörg (Hrsg.) (2005): Ikonologie des Performativen. München: Wilhelm Fink.

[51] Yee Nick (2007): The Proteus Effect: The Effect of Transformed Self-Representation on Behavior. Online::http://vhil.stanford.edu/pubs/2007/yee-proteus-effect.pdf [19.10.2007].

[52]Benjamin Jorissen A Structural Ethnography of Virtual Worlds. In: New Digital Cultural and Educational Spaces ((translated from German)

\title{
Benjamin Jörissen. Strukturale Ethnografie Virtueller Welten (translated from Deutsche)
}

\author{
A.N. Lipov \\ Institute of Philosophy RAS, Russian Federation
}

\begin{abstract}
The existence of so-called «virtual worlds» has been widely known since a series of media publications about the virtual digital platform «Second Life». The publication, which is a translation of a scientific article from German, attempts to analyze the complex mechanisms of creation, distribution, functioning and development in culture and online communities, virtual digital spaces of the latest digital technologies that allow to design high-tech virtual environments and software products with their subsequent introduction and assimilation into the public environment, creating a hybrid virtual-real economy, a new map of social worlds and virtual-social arenas of «real life».
\end{abstract}

Keywords: virtual digital culture, virtual environment, virtual worlds, online communities, structural ethnography, «Second Life» virtual platform, «Avatar» technology, programmed digital objects, interactive algorithms, «Proteus effect», digital public environment and functionality. 